\title{
2. Notation and Conventions.
}

Groups $G$ will always aet on the left. If $M$ is a $G$-module and $\sigma \in G$, this action will be written either as $\mathrm{m} \longmapsto \sigma(\mathrm{m})$ or $\mathrm{m} \longmapsto{ }^{\sigma} \mathrm{m}$. We let $\mathrm{M}^{\mathrm{G}}$ denote the sub-module of $G$-invariants. If $M$ and $N$ are $G$-modules, so is $\operatorname{Hom}(M, N)$ : the action of $\sigma$ on a homomorphism $f: M \longrightarrow N$ is given by

$$
\sigma(f)(m)=\sigma\left(f\left(\sigma^{-1} m\right)\right)
$$

Rings will also act on the left. If $M$ is an R-module and $r \in R$ we let $M_{r}=\{m \varepsilon M: r m=0\}$ denote the sub-module of "r-torsion."

If $A$ and $B$ are elliptic curves (or, more generally, abelian varieties) defined over the field $F$, we let $\operatorname{Hom}_{F}(A, B)$ be the group of algebraic homonorphisms $\phi: A \longrightarrow B$ which are defined over $F$. If $S$ is any F-algebra, we let $A(S)$ denote the abelian group of all S-rational points of A. If $\sigma$ is any automorphism of $F$ we write $\sigma_{A}$ and $\sigma_{B}$ for the conjugate varieties, and $\sigma_{\phi}$ for the conjugate homomorphism from $\sigma_{A}$ to $\sigma_{B}$.

If $F$ is a field, $\bar{F}$ denotes an algebraic closure of it. We shall always use the isomorphism of local class field theory which takes a uniformizing parameter to an arithmetic Frobenius in the Galois group. 